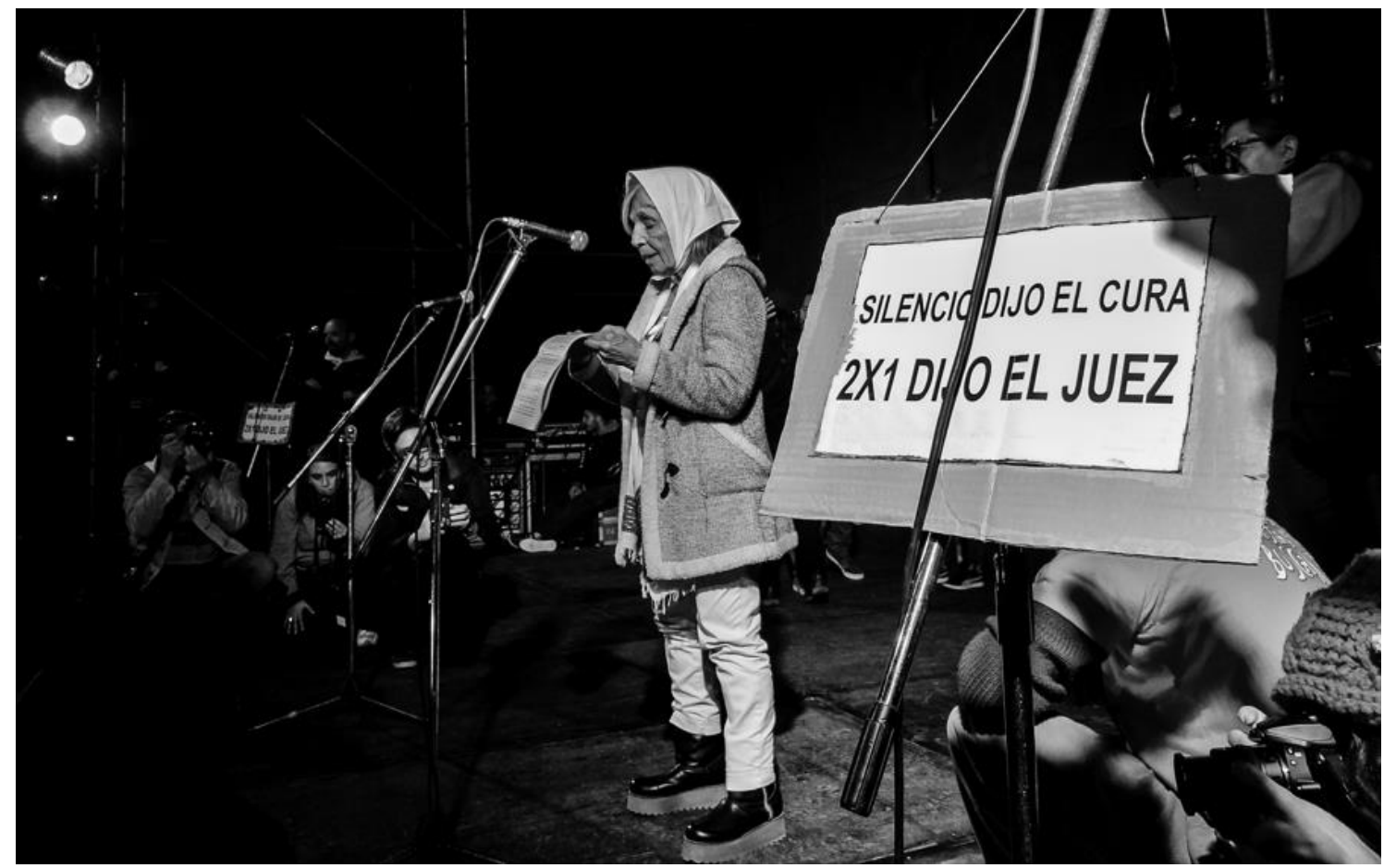

FOTOS: Irina Morán - Revista Alfilo - Periodista Feminista. Militante de Ni Una Menos Córdoba y Mujeres por un parto Respetado 


\title{
LA BRECHA DE GÉNERO EN LA ACADEMIA UNIVERSITARIA: INEQUIDAD DE OPORTUNIDADES DE PARTICIPACIÓN, CAPACITACIÓN Y PROMOCIÓN
}

\author{
María Ángeles Martínez Ruiz ${ }^{1}$ \\ Gladys Merma-Molina ${ }^{2}$ \\ María Alejandra Ávalos Ramos ${ }^{3}$
}

Resumen: Diferentes investigaciones muestran que el incremento cuantitativo de la presencia de la mujer en la universidad puede convertirse en una densa cortina de humo que impide distinguir las micropolíticas de dominación patriarcal. El objetivo de este estudio es desentrañar los mecanismos encubiertos de discriminación que vivencian las mujeres en la universidad. Los resultados muestran que persiste el efecto tijera en las universidades españolas, donde a pesar de acceder más mujeres que hombres a la universidad, ellas descienden abruptamente en las plazas de personal investigador o de catedráticos. La falta de participación se ve agravada por otros sesgos, como el sexismo benevolente, relacionado con el acceso a los espacios de poder. Nuestra propuesta a esta problemática se basa en la necesidad de mantener espacios de participación, sensibles a la diferencia, comunidades de aprendizaje laboral y de práctica profesional que permitan a las académicas participar activamente en la universidad.

Palabras clave: Brecha de género. Igualdad. Ciencia y tecnología.

\section{THE GENDER GAP IN UNIVERSITY ACADEMIA: INEQUITY OF OPPORTUNITIES FOR PARTICIPATION, TRAINING AND PROMOTION}

\begin{abstract}
Different researches show that the quantitative increase of the presence of women in the university can become a dense smokescreen that prevents to distinguish the micropolitics of patriarchal domination. The aim of this study is to unravel the hidden mechanisms of discrimination experienced by women at university. The results show that the scissors effect persists in Spanish universities, where despite the fact that more women than men enter university, they descend abruptly in the positions of research staff or professors. The lack of participation is aggravated by other biases, such as benevolent sexism, related to access to spaces of power. Our proposal to this problem is based on the need to maintain spaces of participation, sensitive to difference, communities of learning at work and professional practice that allow academic women to actively participate in the university.
\end{abstract}

Keywords: Gender gap. Equality. Science and technology.

\section{INTRODUCCIÓN}

La realidad estadística sobre los diferenciales de género es contundente en todos los ámbitos, económico, laboral, y social, ofreciendo un panorama desalentador. Los organismos internacionales como la Organización de las Naciones Unidas, la Organización de las Naciones Unidas para la Educación, la Ciencia y la Cultura, la Comunidad Europea, el Word Bank Group, la Organización para la Cooperación y el

\footnotetext{
${ }^{1}$ Departamento de Didáctica General y Didácticas Específicas - Facultad de Educación, Universidad de Alicante.

${ }^{2}$ Departamento de Didáctica General y Didácticas Específicas - Facultad de Educación, Universidad de Alicante.

${ }^{3}$ Departamento de Didáctica General y Didácticas Específicas - Facultad de Educación, Universidad de Alicante.
} 
Desarrollo Económicos, entre otros, nos alertan continuamente de ello (ONU, 2016; OECD, 2015). En teoría, los gobiernos y las instituciones en la mayoría de países intenta avanzar en políticas de género más consideras e igualitarias. No obstante, la distancia es abismal entre países, por ejemplo entre los países escandinavos y los países del Este y Sur de Europa. El nivel de inequidad de genero en un determinado país puede minar la resistencia de las mujeres en algún momento de su carrera o su vida laboral.

En el campo de la educación, los estudios estadísticos y científicos muestran los altos diferenciales de género en las universidades a favor de los académicos (CIENTÍFICAS EN CIFRAS, 2015; DEPARTMENT OF EDUCATION, 2015; EUROPEAN COMMISSION, 2012; MINISTERIO DE EDUCACIÓN, CULTURA Y DEPORTE, 2015; WORLD BANK, 2011). A pesar de los avances legislativos, políticos y sociales que se han introducido sobre la cuestión de género, las universidades continúan sin conseguir ofrecer plena igualdad de oportunidades a las académicas, especialmente, en lo que se refiere a oportunidades de participación, formación y promoción en la gestión y la investigación.

Aunque las cifras de acceso de las alumnas a la Educación Superior son elocuentes, pueden convertirse en una espesa cortina de humo que impide distinguir las micropolíticas de dominación patriarcal, imperantes todavía en la academia. En base a este marco, el objetivo de este estudio es desentrañar los mecanismos encubiertos de discriminación que vivencian las mujeres en la universidad.

La Gráfica 1 muestra el efecto tijera que se produce en las universidades públicas españolas donde a pesar de acceder más mujeres que hombres a la universidad, tras el grado de doctor, las mujeres descienden abruptamente en la ocupación de plazas de personal docente e investigador, desde las plazas de ayudante (Grado D) hasta las plazas de catedráticos de universidad (Grado A). La Gráfica 2, por su parte, muestra la infrarrepresentación de la mujer en los cargos académicos de las universidades españolas y la Gráfica 3 evidencia la falta de presencia de las mujeres en el Centro Superior de Investigación Científica de España-CSIC. 
GRÁFICA 1 - Distribución de mujeres y hombres a lo largo de la carrera investigadora en universidades públicas. Curso 2014-2015.

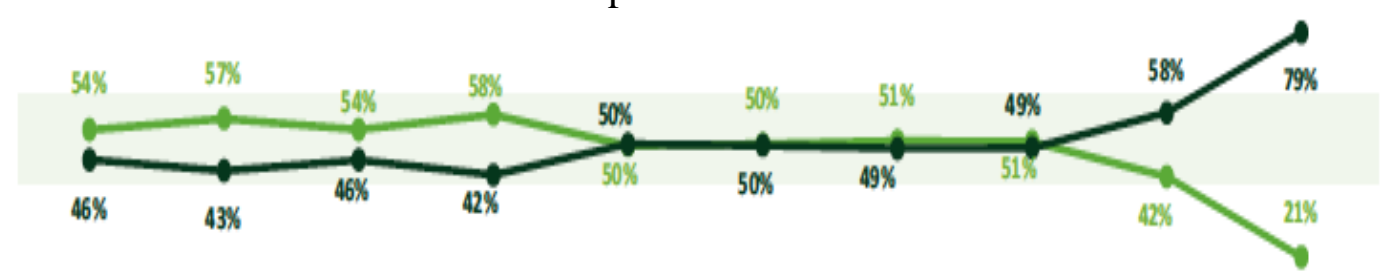

Alumnado Alumnado
matriculado en egresado en matriculado en
estudios de estudios de mádo de matriculado en aprobadas
gradoy 18 y 28 grado 18 y 28
Ciclo.
Ciclo.

emujeres antombres

Fuente: S.G. Coordinación y Seguimiento universitario. Sistema Integrado de Información Universitaria. Ministerio de Educación, Cultura y Deporte.

GRÁFICA 2 - Distribución de mujeres y hombres en órganos unipersonales de gobierno de las universidades públicas, según tipo de órgano, 2015

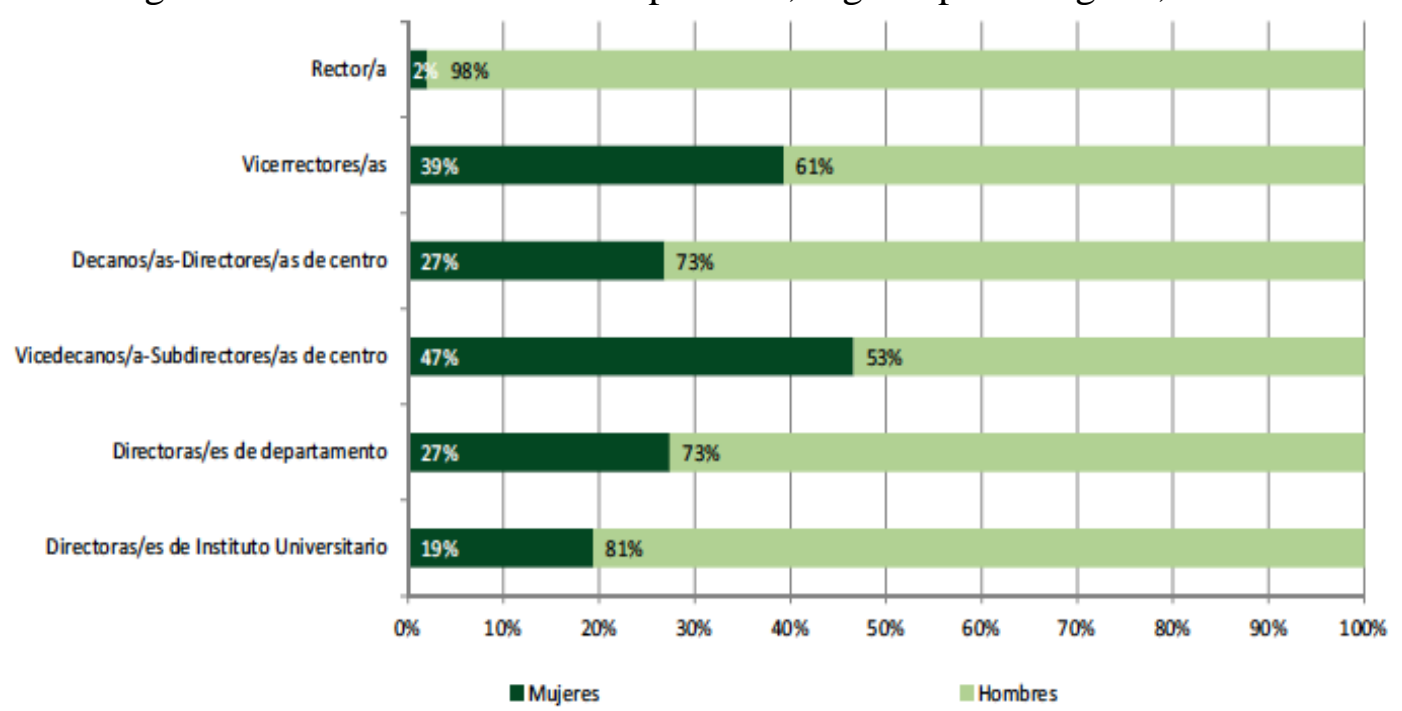

Fuente: S.G. Coordinación y Seguimiento universitario. Sistema Integrado de Información Universitaria. 


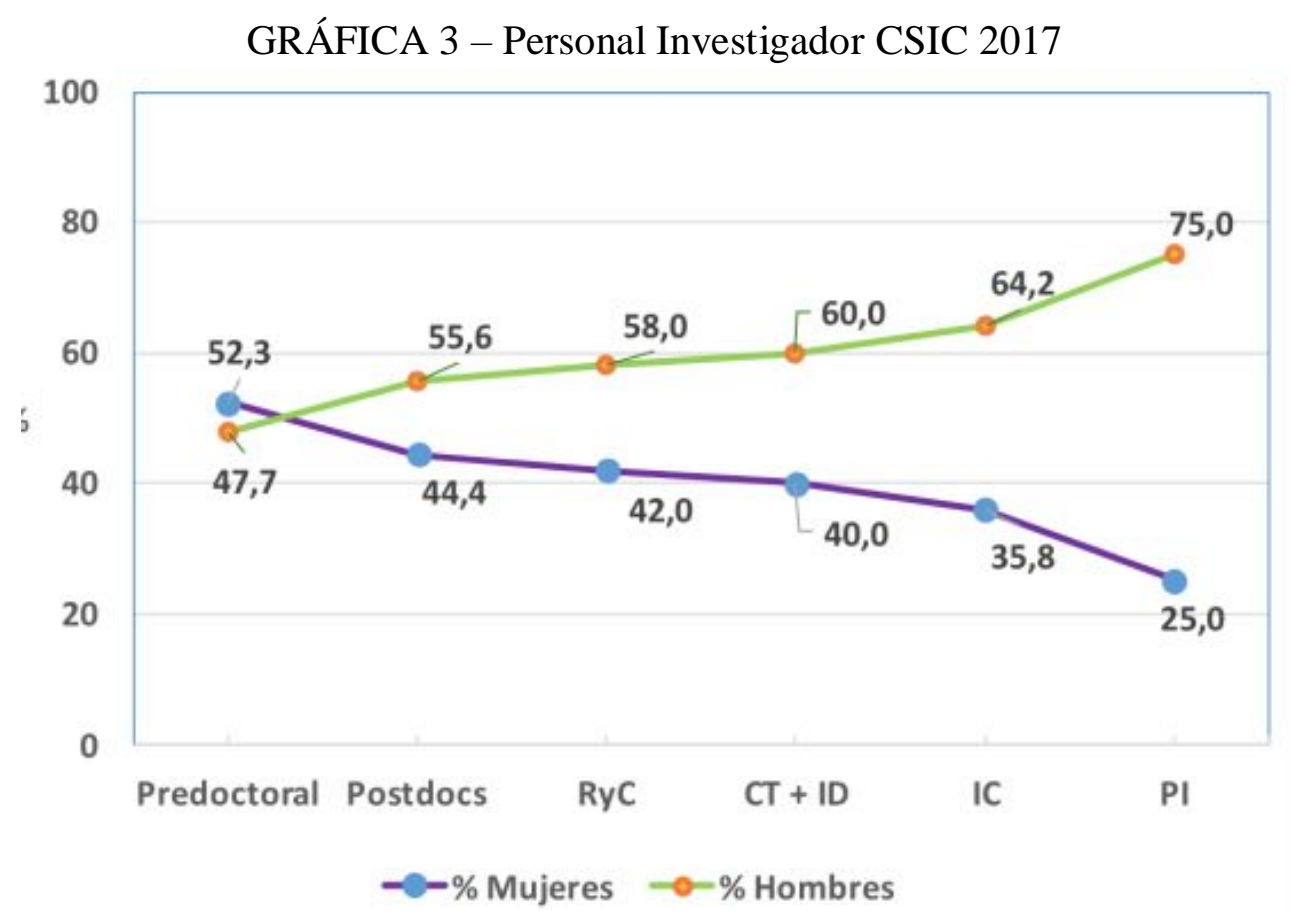

Fuente: Ministerio de Ciencia, Innovación y Universidades

Debido a los altos diferenciales, en muchas instituciones universitarias existe actualmente un compromiso con la paridad en los gobiernos, y se están desarrollando a marchas aceleradas abundantes normativas y políticas de género. En la práctica, hay mucha menos celeridad que en la teoría, por las problemáticas derivadas de los usos sociales tradicionales. Es difícil regular las cuestiones de género con normativas emanadas de los órganos de gobierno universitarios, incluso a veces resulta contraproducente. Es prioritario un esfuerzo de sensibilización a nivel de actitudes y disposiciones personales; quizá esta sensibilización no se ha sido tan efectiva porque los medios de comunicación, en todos los países, siguen dando una imagen debilitada e invisible, cuando no peyorativa, de la mujer en todas los campos sociales.

La exclusión de género en la participación institucional es la fuente de los diferenciales en investigación y promoción. Las oportunidades de aprendizaje y la identidad profesional van ligadas a las posibilidades de participación en las comunidades de conocimiento (Zeichner; Cochran-Smith, 2005). El aprendizaje profesional se sitúa en los espacios de práctica y experiencia, donde se construye el saber de manera negociada y en colaboración con los iguales de una comunidad profesional, por tanto la mengua de oportunidades de participar supone la disminución 
del desarrollo de capacidades personales y profesionales. Por ello, es necesario una cultura del desarrollo profesional del que no estén excluidas las académicas. La universidad, como espacio de relaciones científicas, y como ambiente de profesionalización e investigación, propicia la interacción de sus miembros en busca del conocimiento y la innovación. Disfrutar de un papel activo y asumir responsabilidades en la comunidad de profesionales resulta clave para la formación y la consolidación de la identidad de cada uno de sus integrantes, ya que la merma de oportunidades de participación disminuye las expectativas profesionales. Si una parte de sus integrantes ve menguada su oportunidad de participar, quedará en los márgenes de la comunidad de conocimiento, viéndose limitadas sus potencialidades de desarrollo y del acceso a una competente vida profesional (Lieberman; Pointer-Mace, 2010; Whitcomb; Borko; Liston, 2009). Por el contrario, las oportunidades que la institución ofrezca para el desarrollo profesional de todos y todas serán fundamentales (Vescio; Ross; Adams, 2008).

La escasez de equidad de género en el acceso a la participación formal e informal en los departamentos y grupos de investigación puede resultar nociva, ya que la falta de habilidades y conocimientos adquiridos a través de estas experiencias obstaculiza el avance y progresión de las mujeres en su carrera. Además, en el caso de las académicas, la falta de participación puede verse agravada por otros sesgos implícitos, como el sexismo benevolente (King et al., 2012), relacionado con el acceso a los espacios de poder. En este sentido, también conviene tener presente que las académicas son susceptibles de padecer prácticas de dominación masculina, propias de la cultura del puesto de trabajo (Acker; Dillabough, 2007). A través de los obstáculos de participación, la institución priva a la mujeres del reconocimiento de sus competencias y la oportunidad de desarrollar sus capacidades y su potencial al tiempo que reduce su confianza y seguridad en sí mismas.

Del mismo modo, la participación exige un cambio en la percepción, actitudes y creencias de las mismas académicas. Diferentes estudios manifiestan una gran distancia entre la auto-confianza en los distintos géneros. Así, Kay y Shipman (2014) evidencian que, a diferencia de los hombres, un gran número de mujeres a pesar de estar bien situadas profesionalmente presenta bajos niveles de confianza en sus capacidades y tienden a infravalorar sus propios logros. Estos sentimientos de inferioridad tienen 
efectos devastadores en su nivel de iniciativa y participación, ya que la seguridad en sí mismas y en la propia imagen constituyen condiciones indispensables para elevar las aspiraciones, lanzarse hacia adelante y actuar. Por tanto, la exclusión de las académicas en la universidad no se debe únicamente a factores externos de desigualdad, como las prácticas discriminatorias de la propia institución, sino también a las limitaciones autoimpuestas por ellas mismas. En esta línea, resuenan las ideas de Sandberg (2013), que argumenta que es la propia mujer quien se impone a sí misma obstáculos para participar, muchas veces por la falta de autoconfianza, otras por bajas expectativas y otras por el temor a ser percibidas como pretenciosas o ambiciosas. Algunos estudios evidencian que la mujer ha sido educada más para agradar, que solicitar el respeto y las oportunidades de participación que merece (Kay; Shipman, 2014). Asimismo, el miedo al fracaso, y la falta de seguridad, le impide la toma de decisiones rápidas cuando son necesarias. En general, tiende a culpabilizarse en exceso por los errores y su preocupación por la excelencia suele frenar su actuación. Una expectativa siempre por debajo de sus logros, le llevan a rechazar las oportunidades en campos en los que es ampliamente competente (Sandberg, 2013); no obstante, la inseguridad, la devaluación y la falta de confianza, que silencian a la mujer y limitan sus oportunidades de participar en la comunidad, se pueden superar modificando los elementos que inciden en la visibilidad y con la inserción en autenticas redes de apoyo y colaboración.

En suma, las académicas tienen derecho a disfrutar de idénticas oportunidades de participación y de liderazgo que sus colegas, no podemos consentir que obstáculos externos ni condicionantes de origen interno dificulten su derecho a participar, actuar y decidir (Genset, 2010).

En cualquier caso, la batalla por la igualdad la deben dar y ganar las propias mujeres. En la Universidad de Alicante, el Centro de la Mujer (CEM) fue el que posibilitó la unión de las académicas más concienciadas y activista y supuso el inicio de un movimiento de base en la década de los 90. Actualmente, y a menudo, no se percibe tanto la fuerza de los inicios. Luchar por la igualdad de forma sostenida exige renunciar a una parcela cómoda y trasladarse a un campo de esfuerzo, coraje y reivindicación. Exige estar pendiente, en el día a día, de las microdesigualdades que sufren las mujeres. Es cierto que no tenemos muchos modelos a seguir que sean mujeres pero hay que 
participar, elevar nuestra voz, rendir en el trabajo, competir, exigirnos a nosotras mismas.

En el campo universitario, hemos recogido en diversos estudios las voces de las academias de nuestra universidad (Lozano; Iglesias; Martínez, 2014; Martínez; Mañas, 2016). En estos estudios hemos encontrado voces y visiones de las académicas que han sido activista en las iniciales causas de género en la universidad. En su etapa inicial, estas académicas han alertado a la comunidad sobre las cuestiones de género, que están ahí de forma cotidiana y permanente, agazapadas, que forman parte de nuestra cultura impidiéndonos salir de una universidad patriarcal y paternalista. En una estructura tan sólida, consolidada y jerarquizada como la universitaria, similar al ejército, la iglesia, o las empresas donde las potentes estructuras de poder patriarcal y la negociación de género depende del poder establecido durante generaciones, no podemos esperar que quieran ceder sus derechos sin más, cuando el poder sigue siendo masculino. La equidad de género no es un acto de retorica es un acto político y social de riesgo contra un arraigado status quo.

Es cierto que la derecha y la izquierda política han asimilado un lenguaje correcto, pero sin un cambio social profundo, se edifica y se desmantela al mismo tiempo, en un proceso de involución y balanceo. En parte, muy conscientemente en los sectores de poder, se utiliza a la mujer pero no se la escucha. Y la universidad no es ajena a lo que pasa en la sociedad; es decir, en la academia encontramos acoso; y eso sigue estando ahí como en el resto de la sociedad. Pero la universidad es reacia al escandalo y siempre ha procurado alejar a los acosadores por un tiempo, incumpliendo determinadas normativas de manera flagrante.

Frente a estos contextos políticos hay que permanecer continuamente alertas y comprometidas, día a día. No se puede ir hacia atrás, ni parar, porque en cuanto una se relaja, desaparece, y vuelve la invisibilidad más absoluta. Las académicas activistas que han tenido un compromiso de género han realizado un trabajo generoso, a base de compromiso y sacrificio, persistencia e insistencia, y ello produce cansancio y desaliento, por ello, debemos estar muy agradecidas.

En un segundo momento, podemos percibir que la institucionalización de lo políticamente correcto en género en las universidades, sin duda le ha quitado fuerza de combate al movimiento de académicas activistas. El movimiento se ha hecho menos 
crítico, y más dependiente del poder. Las instituciones tratan de crear estructuras cómodas para los distintos equipos de gobierno de las universidades, de tal manera que, como suele pasar en política, se dotan Unidades de Igualdad, salvo honrosas excepciones, con personas que no tienen ni experiencia, ni trayectoria en el activismo de género, personas que no sean incómodas. Porque el feminismo es muy incómodo, y muy irreverente, con el paternalismo. Se crean escenarios donde hay un bonito espacio que dice Igualdad, pero en realidad detrás no hay nada. También existe un sentir de que las concepciones y la cultura no llega a cambiar y las reformas no calan en la mentalidad institucional en muchos casos. Si se analiza el discurso, si se analizan las concepciones latentes en los círculos de poder, sutilmente salta el machismo; por tanto, solo existe una mentalidad aparente y es esta la que se ve en la superficie. Ahora sería políticamente incorrecto estar en contra; es decir, que la apariencia se ha normalizado y algunas cosas también se han normalizado como la paridad y la conciliación.

No obstante, en muchas activistas, existe un cierto sentimiento de desilusión, de escepticismo, de cuestionarse si la unidad de igualdad, como la unidad de calidad, existen pero no cambian la realidad. Seguimos mirando a otro lado, y se ha perdido el poder emancipador y el poder combativo de los inicios.

$\mathrm{Y}$ en la realidad y en las concepciones latentes de la cultura universitaria queda mucha labor por hacer. Por ello, hay participantes que pese al desencanto siguen dispuestas al compromiso y la lucha por sus visiones: las mujeres tenemos que ir consolidando los derechos día a día, y no se debe retroceder.

En síntesis, las académicas participantes en nuestros estudios (Lozano; Iglesias; Martínez, 2014; Martínez; Mañas, 2016) perciben la permanencia de concepciones latentes negativas, estiman que hay una tendencia a crear estructuras cómodas para el poder y contemplan que los cambios no han afectado a los diferenciales estadísticos. En su opinión, sigue existiendo una necesidad de mantener el compromiso, sigue existiendo la urgencia de avanzar en las acciones de género, en visibilizar los diferenciales de género estadísticos y prácticos, hasta que se salvaguarden y se garanticen los derechos de las mujeres a obtener las mismas oportunidades que los varones en base a sus competencias profesionales (Stone, 2013). Por su lucha, la fuerza de su voluntad, la profesionalidad, el coraje y la pasión en su tarea las académicas activistas en la Universidad de Alicante (España) merecen pleno reconocimiento. 


\section{DISCUSIÓN Y CONCLUSIONES}

Los obstáculos en la promoción (Dugger, 2001), la desigualdad en las condiciones laborales (Bagilhole; Goode, 2001; Meulders; Plasman; Rigo; O'dorchai, 2010), la falta de apoyos y de referentes femeninos (Ragins; Cotton, 1991), así como las dificultades en la participación y las limitaciones autoimpuestas (Kay; Shipman, 2014; Knights; Richards, 2003) son barreras que las mujeres han de superar conjuntamente, a través de la creación de una masa crítica que vaya habitando los espacios de conocimiento.

En las últimas décadas, son numerosas las investigaciones que han mostrado cómo las fórmulas tradicionales de exclusión han evolucionado hacia un sistema más complejo y sofisticado (Bevan; Learmonth, 2012; Morley, 2006; Stromquist, 2015). Como espacio de poder simbólico, el sistema patriarcal ha adoptado nuevas formas de dominación mediante las prácticas y las relaciones cotidianas que se generan en la academia. En las aulas, los hombres se apropian del discurso y tienden a establecer un número más alto de interacciones con el profesorado (Mosconi, 2001), quien a su vez tiende a reforzar este mayor protagonismo (Davis, 2003). A ello se ha de añadir el sexismo lingüístico, que viene a agravar la invisibilización de la mujer (Kleinman, 2002). El género se construye en el diálogo social y el carácter universal del discurso que oculta el predominio de la masculinidad hegemónica (Parks; Roberton, 2005; Wright; King, 1990). Los comentarios despectivos, las bromas sexistas y el lenguaje paternalista forman también parte de la cultura implícita de exclusión. Asimismo, se han de considerar los sesgos sexistas que influyen en la evaluación de los aprendizajes de las alumnas y de los alumnos, especialmente en aquellos campos de conocimiento tradicionalmente ocupados por los hombres, tales como la ciencia, la tecnología, la ingeniería o las matemáticas (Riegle-Crumb; Humphries, 2012; Xie; Fang; Shauman, 2015). No considerarlas hábiles en estos ámbitos provoca la infravaloración de su esfuerzo y trabajo y, en última instancia, la disminución de su confianza y seguridad personal (Cech; Rubineau; Silbey; Serond, 2011). Incluso en aquellos casos en los que se evalúan adecuadamente sus logros académicos, estos siempre son atribuidos a cualidades menos valoradas que las que provocan el éxito masculino (Morley, 2006). 
El carácter intangible y sutil de estos mecanismos de exclusión en los espacios universitarios - departamentos, grupos de investigación, facultades -, contribuye a la neutralización de la inequidad, a diluirla de forma que su detección es menos factible. El estudio de Morrison, Bourke y Kelley (2005) presenta las dificultades que existen a la hora de identificar y reconocer la existencia de estas prácticas discriminatorias, también por parte de quienes las padecen. Socializadas en los estereotipos tradicionales de género, las alumnas y profesoras suelen considerar la universidad como un espacio de plena igualdad entre mujeres y hombres, negando la existencia de discriminación (Francis; Burke; Read, 2014). Incluso cuando son capaces de apreciar ciertos comportamientos sexistas, se impone la idea de lo irrelevante (Beagan, 2001). En estos términos se expresa también Morley (2006), cuando nos advierte del peligroso efecto que estas desigualdades cotidianas provocan en la construcción de la identidad académica y profesional de la mujer, especialmente cuando ocurren en espacios políticamente correctos como es la universidad.

Nuestra propuesta al problema de la participación de la mujer en la vida universitaria es la necesidad de mantener espacios de participación, sensibles a la diferencia. Un espacio de comunidades de aprendizaje laboral y de práctica profesional que permita a las académicas integrarse y participar a nivel institucional como sus compañeros (Kurtz-Costes; Andrews-Helmke; Ülkü-Steiner, 2006; Nap, 2009). Los espacios de participación aportan confianza, visibilidad, es decir reconfortan. Son un punto de referencia para inquietudes, para a impulsar una cultura institucional sensible a las microdesigualdades que cotidianamente minan las oportunidades de género (Maher; Tetreault, 2011). Los departamentos y grupos de investigación no pueden desperdiciar el potencial de las académicas y deben procurar su completa inserción en las tareas académicas, tanto ofreciéndoles oportunidades de mentoría como a través del trabajo de pares. No dejar ninguna académica atrás supone eliminar preconcepciones y barreras. En este espacio la académica debe esforzarse al máximo y no dejar que sus roles tradicionales de mujer le impidan cumplir sus proyectos profesionales.

\section{REFERENCIAS}


Acker, Sandra; Dillabough, Jo-Anne. (2007). Women 'learning to labour' in the 'male emporium': Exploring gendered work in teacher education. Gender and Education, 19, (3), pp. 297-316.

Bagilhole, Bárbara; Goode, Jackie. (2001). The contradiction of the myth of individual merit, and the reality of a patriarchal support system in academic careers: A feminist investigation. European Journal of Women's Studies, 8(2), pp. 161-180.

Beagan, Brenda. (2001). Micro inequities and everyday inequalities: Race, gender, sexuality and class in medical school. The Canadian Journal of Sociology, 26(4), pp.583-610.

Bevan, Valerie; Learmonth, Mark. (2012). I wouldn't say it's sexism, except that ... It's all these little subtle things: Healthcare scientists' accounts of gender in healthcare science laboratories. Social Studies of Science, 43(1), pp. 136-158.

Cech, Erin; Rubineau, Brian; Silbey, Susan; Serond, Caroll. (2011). Professional role confidence and gendered persistence in engineering. American Sociological Review, 76(5), pp. 641-666.

\section{CONSEJO SUPERIOR DE INVESTIGACIONES CIENTÍFICAS-CSIC. Informe mujeres investigadoras, 2018.}

Davis, Kathryn. (2003). Teaching for gender equity in physical education: a review of the literature. Women in Sport \& Physical Activity Journal, 12(2), pp. 55-81.

\section{DEPARTMENT OF EDUCATION, NATIONAL CENTRE FOR EDUCATION}

STATISTICS. The condition of education, 2015. Recuperado de:

http://nces.ed.gov/pubs2015/2015144.pdf

Dugger, Karen. (2001). Women in Higher Education in the United States: I has there been progress?. International Journal of Sociology and Social Policy, 21( 1/2), pp. 118130.

EUROPEAN COMMISSION. She Figures 2012. Gender in Research and Innovation Statistics and Indicators. Luxembourg: Office for Official Publications of the European Communities, 2012. Recuperado de: http://ec.europa.eu/research/sciencesociety/document library/pdf 06/she-figures-2012 en.pdf

Francis, Becky; BURKE, Penny; READ, Bárbara. (2014). The submergence and reemergence of gender in undergraduate accounts of university experience. Gender and Education, 26(1), pp. 1-17.

Genset. Recomandation for action on the gender dimension in Science, 2010. Recuperado de: http://www.genderinscience.org/downloads/genSETConsensusReportRecomendationsf orActiontheGenderDimensioninScience.pdf. 
Kay, Katty; Shipman, Claire. (2014). The confidence code. The science and art of selfassurance - what women should know. New York: Harper Collins Publishers.

King, Eden; Botsford, W; Helb, MR; Kazama, S; Dawson, JF; Perkins, A. (2012) Benevolent sexism at work: Gender difference in the distribution of challenging developmental experiences. Journal of Managament, 38(6), pp. 1835-1866.

Kleinman, Sherryl. (2002). Why sexist language matters. Qualitative Sociology, 2(2), pp. 299-304.

Knights, David; Richards, Wendy. (2003). Sex discrimination in UK academia. Gender, Work and Organization, 10(2), pp. 213-237.

Kurtz-Costes, Beth; Andrews Helmke, Laura; Ülkü-Steiner, Beril. (2006) Gender and doctoral studies: The perceptions of Ph. D. students in an American university. Gender and Education, 18(2), pp. 137-155.

Lieberman, Ann y Pointer Mace, Desiree. (2010). Making practice public: Teacher learning in the 21st century. Journal of Teacher Education, 61(1-2), pp. 77-88.

Lozano, Inés, Iglesias, Marcos; Martínez, María Ángeles. (2014). Las oportunidades de las académicas en el desarrollo profesional docente universitario: Un estudio cualitativo. EducaciónXX1, 17(1), pp.157-182.

Maher, Frances; Tetreault, Mary Kay Thompson. (2011). Long-term transformations: excavating privilege and diversity in the academy. Gender and Education, 23(3), pp. 281-297.

Martínez, María Ángeles; Mañas, Carmen. El inicio de la visibilidad: el centro de Estudios de la Mujer de la Universidad de Alicante. In M. Álvarez, R. Bohórquez, J.C. Velásquez. (Coord.). (2016). Diseño y Atención a las oportunidades de género en la Educación Superior (pp. 34-56). Bogotá, DC: Editorial FEDICOR.

Meulders, Danièle; Plasman, Robert; Rigo, Audrey; O’dorchai, Sile. (2010). Horizontal and vertical segregation. Meta-analysis of gender and science research-topic report. Recuperado de: https://genderedinnovations.stanford.edu/images/TR1_Segregation.pdf

MINISTERIO DE EDUCACIÓN, CULTURA Y DEPORTE (MECD). Datos y cifras del sistema universitario español. Curso 2014/2015. Madrid: Subdirección General de Documentación y Publicaciones del Ministerio de Educación, Cultura y Deporte, 2015. Recuperado de: http://www.mecd.gob.es/dms/mecd/educacion-mecd/areaseducacion/universidades/estadisticas-informes/datos-cifras/Datos-y-Cifras-del-SUECurso-2014-2015.pdf

Morley, Louise. (2006). Hidden transcripts: the micropolitics of gender in Commonwealth universities. Women's Studies International Forum, 29, pp.543-551. 
Morrison, Zoe; Bourke, Mary; Kelley, Caroline. (2005). Stop making it such a big issue: Perceptions and experiences of gender inequality by undergraduates at a British University. Women's Studies International Forum, 28, pp. 150-162.

Mosconi, Nicole. (2001). How do they make the teaching practices of gender inequality? Records of Sciences of Education, 5, pp. 97-109.

NAP-The National Academy Press. (2009). Gender differences at critical transitions in the careers of science, engineering and mathematics faculty. Washington, DC: The National Academies Press.

OECD. (2015). Report to G7 Leaders on women and entrepreneurship.

ONU-MUJERES. (2016). Hechos y cifras: Acabar con la violencia contra mujeres y niñas.

Parks, Janet; ROBERTON, Mary Ann. (2005). Explaining age and gender effect on attitudes toward sexist language. Journal of Language and Social Psychology, 24(4), pp. 401-411.

Ragins, Belle Rose; Cotton, John. (1991). Easier said than done: Gender differences in perceived barriers to gaining a mentor. Academy of Management Journal, 34(4), pp.939-951.

Riegle-Crumb, Catherine; Humphries, Melissa. Exploring bias in Math teachers' perceptions of students' ability by gender and race/ethnicity. Gender \& Society, 26(2), pp. 290-322, 2012.

Sandberg, Sheryl. (2013). Lean in: Women, work, and the will to lead. Random House.

Stone, Pamela. (2013). “OPTING OUT” Challenging Stereotypes and Creating Real Options for Women in the Professions. Recuperado de: https://www.hbs.edu/faculty/conferences/2013-w50-researchsymposium/Documents/stone.pdf.

Stromquist, Nelly. (2015). Women in Higher Education today -structure and agency from a gender perspective. Journal of Educational Planning and Administration, 29(3), pp. 287-306.

UNIDAD DE MUJERES Y CIENCIA-UMYC. (2016). Científicas en cifras 2015. Estadísticas e indicadores de la (des) igualdad de género en la formación y profesión científica.

UNITED NATIONS. (2015). Transforming our world: The 2030 agenda for sustainable development. Resolution adopted by the General Assembly. 
Vescio, Vicki; Ross, Dorene; Adams, Alyson. (2008). A review of research on the impact of professional learning communities on teaching practice and student learning. Teaching and Teacher Education, 24, pp. 80-91.

Whitcomb, Jennie, Borko, Hilda; Liston, Dan. (2009.). Growing talent: Promising professional development models and practices. Journal of Teacher Education, 60(3), pp. 207-212.

World Bank. (2011). World development report 2012: Gender equality and development. Washington, DC: World Bank.

Wright, Jan; King, Richard. (1990). I say what I mean," said Alice: an analysis of gendered discourse in Physical Education. Journal of Teaching in Physical Education, 10(2), pp. 210-225.

Xie, Yu; Fang, Michael; Shauman, Kimberlee. (2015). STEM Education. The Annual Review of Sociology, 41, pp.331-357.

Zeichner, Kenneth; Cochran-Smith, Marilyn. (2005). Studying teacher education: The report of the AERA panel on research and teacher education. Lawrence Erlbaum Associates. 